\title{
Selection of solitary waves in vertically falling liquid films
}

\author{
N. Kofman ${ }^{\mathrm{a}}$, C. Ruyer-Quil ${ }^{\mathrm{b}}, \mathrm{S}$. Mergui $^{\mathrm{c}}$ \\ ${ }^{a}$ Univ Paris-Sud, CNRS, Lab FAST, 91405 Orsay, France. Present address : EPFL-LFMI, Station 9, 1015 Lausanne, Suisse - nicolas.kofman@epfl.ch \\ ${ }^{b}$ Univ Savoie Mont Blanc, CNRS, Lab LOCIE, 73000 Chambéry, France - christian.ruyer-quil@univ-smb.fr \\ ${ }^{c}$ Univ Paris-Sud, CNRS, Lab FAST, 91405 Orsay, France - mergui@fast.u-psud.fr
}

\begin{abstract}
Two-dimensional solitary waves at the surface of a film flow down a vertical plane are considered. When the system is subjected to inlet white noise, solitary waves are formed after an inception region and interact with each other. Using open-domain simulations of reduced equation models, we investigate numerically their late time process dynamics. Close to the instability threshold, the waves synchronize themselves into bound states. For higher values of the Reynolds number, the separation distance between the waves increases and the synchronization process at work is weaker. Performing statistics, we show that the mean characteristics of the waves correspond to the minimal value of the mean film thickness along the traveling-wave branch of solutions. In this regime, synchronization occurs through the waves tails which is associated with a change of scaling of the waves features. A similar behaviour is observed performing simulations in periodic domains: the selected waves maximize the mean flow rate.
\end{abstract}

Keywords: thin liquid films, solitary waves, reduced equation models

\section{Introduction}

Falling liquid films are widely used in industrial applications due to their interesting properties regarding heat and mass transfer. Interfacial waves intensify transfers by a factor up to two in comparison to the flat film situation [10], though the exchange surface undergoes an increment of only a few percents [20]. The reason for this paradox lies in the organization of the wavy motion of the film around isolated structures, or solitary waves, interacting one with the other through nearly flat regions of the film. These so-called solitary waves are composed of a main hump, a series of capillary ripples and a flat part called substrate. Whenever the amplitude of these waves is high enough, a recirculation region, i.e. a roll, appears in the main hump [25]: this mixing is a key element to transfer intensification [34]. At given flow conditions, the onset of recirculation regions is sensitive to the separation distance between waves and therefore to the frequency [24]. It is thus essential to determine the preferred wavelength of the natural evolution of the film. This selection process is the subject of the current paper.

The onset of the different regimes of the film dynamics is function of the reduced Reynolds number, denoted $\delta$, and introduced by [28]. This parameter combines inertia, viscosity and surface tension and will be defined in $\S 2.1$. When $\delta<1.5$, inertia effects are weak and the amplitude of the waves remains small (drag-gravity regime). Selection of the waves in this case has been the object of several works, within the framework of the hydrodynamic bound states theory, and based on var- ious equation models: the weakly nonlinear Kawahara equation [16, 22, 32], the Benney equation [9], the Kapitza-Shkadov model [4] and more recently the Ruyer-Quil-Manneville model [23]. A discrete set of preferred wavelengths is observed as waves tend to form bound states through interaction of their tails with the capillary ripples at the front of the next ones. These separation distances correspond to discrete values of stability of the waves with respect to infinitesimal perturbations, and to local minima of the viscous dissipation function along the traveling-wave branch of solitary waves.

When $\delta>1.5$, the amplitude and speed of the waves increase drastically (drag-inertia regime). In that regime, the natural evolution of the waves show some similarities with the inverse energy cascade observed in 2D turbulence [17]. One can observe successively formation of linear waves, their non-linear saturation, evolution towards solitary waves through quasi periodic or subharmonic secondary instabilities, wave merging and increasing of the separation distance between solitary waves. The value to which this separation distance converges is still an open question in the literature. It has been addressed only by Chang et al. [4] but using the Kapitza-Shkadov model and making strong assumptions on the details of the dynamics. Other studies are available in the turbulent case on the coarsening of the so-called roll waves [1].

Eventually, let us introduce the concept of optimal wave which is the main motivation of this study. It is due to Trifonov [30] and corresponds to a minimum of the mean height along the traveling-wave branch of solitary waves. Short and long op- 
timal waves are distinguished, depending on the value of the associated separation distance. We will show in this paper by means of time simulations of the Ruyer-Quil-Manneville model that the long-time dynamics of the film converges to these solutions. Links will be made with results from the linear stability analysis, and with the argument of minimal viscous dissipation.

The paper is organized as follows. Section 2 presents the numerical tools. In section 3, we revisit the literature results in the drag-gravity regime $(\delta>1.5)$. Section 4 presents the results obtained in the drag-inertia regime. Conclusions are given in Section 5 .

\section{Numerical tools}

\subsection{Notations}

We consider a liquid film falling down a vertical plane (Figure 1). The flow is assumed to be Newtonian with constant physical properties (surface tension $\sigma$, viscosity $\mu$, density $\rho$ ). We denote by $v=\mu / \rho$ the kinematic viscosity and $g$ the acceleration of gravity. The current study is carried out in the $2 \mathrm{D}$ case, that is only by considering spanwise invariant waves with $x$ being the coordinate in the direction of the flow and $y$ the coordinate oriented in the normal direction to the plane. $u$ and $v$ refer to the velocity components in the two directions $x$ and $y$.

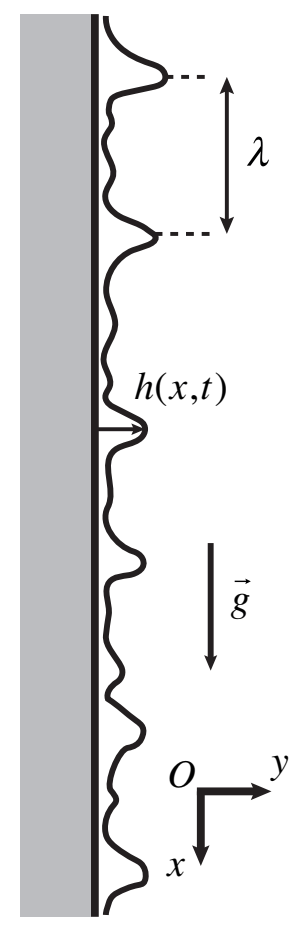

Figure 1: Sketch of the problem set-up

Two length scales can be defined balancing viscosity, acceleration of gravity and surface tension: the capillary length $l_{c}=\sqrt{\sigma / \rho g}$ and the viscous-gravity length $l_{v}=v^{2 / 3} g^{-1 / 3}$. Adding the flat film thickness $\bar{h}_{N}$ (Nusselt thickness) allows to get a first set of non-dimensional parameters. It is composed of the Kapitza number $\Gamma=\left(l_{c} / l_{v}\right)^{2}$ and the Reynolds number $R e=\bar{q}_{N} / v=h_{N}^{3} / 3$ built from the flow rate $\bar{q}_{N}$ or the nondimensional Nusselt thickness $h_{N}=\bar{h}_{N} / l_{v}$.
The Kapitza number takes rather high values in practice which is not convenient from a numerical point of view. This led Shkadov [28] to introduce a specific non-dimensionalization, compressing the $x$ coordinate by a factor $\kappa$ with respect to the $y$ direction. This factor is adjusted to balance the gravity force and the capillary pressure gradient, i.e. $k=\left(l_{c} / \bar{h}_{N}\right)^{2 / 3}$. The Shkadov scaling yields two non-dimensional numbers: the reduced Reynolds number $\delta=3 R e / \kappa$ and a viscous dispersion parameter $\eta=1 / \kappa^{2}$. It sets the coefficient of the capillary pressure term to one and makes explicit the balance of all forces in the equations (gravity, viscosity, surface tension and inertia).

\subsection{WRIBL model}

Hereinafter we will mimic the dynamics of the film based on the solutions to a reduced set of equations involving only two variables: the film thickness $h(x, t)$ and the local liquid flow rate $q(x, t)=\int_{0}^{h} u d y$. This model has been validated through comparisons to DNS and experiments [14] showing excellent agreement for the parameter range of interest. It allows to get access to the essence of the flow dynamics at reasonable computational costs. Let us briefly outline its derivation procedure starting from the governing equations.

First, the long-wavelength nature of the instability enables to invoke a separation of scale between the $x$ and $y$ coordinates. In practice, this is performed by introducing a small parameter $\epsilon=\partial_{x, t}$ and ordering terms into powers of $\epsilon$. Then, the classical boundary-layer approximation is followed: pressure is computed after integration of the $y$-momentum balance where $O\left(\epsilon^{2}\right)$ inertial terms have been dropped out. Depth-averaging of the $x$ momentum equation yields an evolution equation for the flow rate consistent up to $O\left(\epsilon^{2}\right)$. Let us mention that using specific weights through this latter procedure allows considerable algebraic simplification (weighted-residual technique, see [14] for more details). The so-obtained Weighted Residual Integral Boundary Layer model is composed of a (exact) depth-averaged mass-conservation equation and a momentumconservation equation. It is written as:

$$
\begin{gathered}
\partial_{t} h+\partial_{x} q=0 \\
\delta \partial_{t} q=\frac{5}{6} h-\frac{5}{2} \frac{q}{h^{2}}+\delta\left[\frac{9}{7} \frac{q^{2}}{h^{2}} \partial_{x} h-\frac{17}{7} \frac{q}{h} \partial_{x} q\right]+\frac{5}{6} h \partial_{x x x} h \\
+\eta\left[4 \frac{q}{h^{2}}\left(\partial_{x} h\right)^{2}-\frac{9}{2 h} \partial_{x} q \partial_{x} h-6 \frac{q}{h} \partial_{x x} h+\frac{9}{2} \partial_{x x} q\right]
\end{gathered}
$$

As already underlined, this model is consistent up to $O\left(\epsilon^{2}\right)$ with the long-wave expansion and thus adequately accounts for the second-order viscous effects, i.e. the elongational viscous terms (last row of (1b)), which are omitted in most low-dimensional models, for instance the classical Kapitza-Shkadov model [27] as the viscous dispersion parameter $\eta$ is generally small. Yet, the linear stability of the Nusselt base flow is significantly affected by the presence of these terms as they decrease the speed of kinematic waves. We recall here that the imbalance of the kinematic-wave speed with the flow speed at the free surface is the key ingredient for the onset of the instability as pointed by [29] and [14] within the Whitham wave-hierarchy framework [33]. 


\subsection{Two-dimensional traveling-wave solutions}

Traveling wave branches of solution are computed according to the following procedure. The coordinates $x$ and $t$ are combined into $\xi=x-c t$ in the moving frame of reference where $c$ is the wave speed. This allows to integrate the massconservation equation yielding $q=c h+q_{0}$. A single o.d.e. is thus obtained which can be recast in the form of an autonomous dynamical system in a 3D phase space spanned by $h$, and its two derivatives $h_{\xi}$, and $h_{\xi \xi}$.

The constant $q_{0}=\int_{0}^{h}(u-c) d y$ represents the flow rate under the wave in its moving frame. Considering traveling waves, i.e. limit cycles of period $\lambda$ in the phase space, $q_{0}$ is adjusted so that the average $\langle h\rangle_{\xi}=\lambda^{-1} \int_{0}^{\lambda} h d \xi$ corresponds either to the waves generated by the synchronization between the flow and an inlet forcing (see for instance the experimental work by [18]), or to the spatial periodicity of numerical simulations in a periodic domain. In the former case, $\langle q\rangle_{\xi}=1 / 3$, which is the value of the flow rate corresponding to the Nusselt solution of thickness unity. For the latter, the total mass being conserved, we get $\langle h\rangle_{\xi}=1$ [26]. In practice, we have used the software AUTO07p [8] and computed the traveling wave branches of solution by continuation starting from Hopf bifurcation and increasing the period. Let us mention that the traveling waves computed with these two conditions are in fact identical provided that the Reynolds number based on the substrate thickness $h_{s}$ is used. This latter quantity can be obtained by assuming a Nusselt profile in the substrate and solving the following $3^{\text {rd }}$ order polynomial equation: $h_{s}^{3} / 3=c h_{s}+q_{0}$.

\subsection{Time-dependent simulations}

We perform spatio-temporal simulations of the equations model using an algorithm that is briefly described here. Let us write formally the system (1) in the form:

$$
\partial_{t} \mathbf{H}=\mathcal{L}(\mathbf{H})+\mathcal{N}(\mathbf{H})
$$

$\mathbf{H}=(h, q)$ is the vector of unknowns, $\mathcal{L}$ and $\mathcal{N}$ are linear and nonlinear operators, respectively. The code employs the CrankNicholson temporal scheme with first order Taylor expansion of the nonlinear operator. Spatial derivatives are approximated using centered finite differences. The truncation error is thus of second order both in time and space. Two kinds of simulations associated with different boundary conditions have been performed. The first-set of boundary conditions corresponds to an open domain, for which no-reflection conditions are applied at the outlet. This open-flow situation mimics the experimental conditions. The second-set of boundary conditions is the periodic boundary conditions, or closed-flow situation.

In an open domain, the open-flow boundary conditions are written as:

$$
\begin{gathered}
\mathbf{H}(x, 0)=\mathbf{P}(x), \mathbf{H}(0, t)=(1,1 / 3)+\mathbf{B}(t), \\
\partial_{t} \mathbf{H}(L, t)=v_{f} \partial_{x} \mathbf{H}(L, t) .
\end{gathered}
$$

The function $\mathbf{P}(x)$ can be taken as a constant equal to $(1,1 / 3)$. To speed up the transient phase of computations, we have rather used an ad-hoc profile of the form $a_{0}-a_{1} \arctan \left(a_{2} x+a_{3}\right)$ with $\left(a_{i}\right)_{i}$ suitably chosen. The function $\mathbf{B}(t)$ is a temporal white noise. The condition (3b), imposed by dimensionality constraints, is an ad-hoc expression which allows to limit wave reflection at the outlet $\left(v_{f}=1\right.$ typically). In practice, condition (3b) "hyperbolizes" our system of equations at the end of the numerical domain, ensuring that the information travels downward. The drawback of using (3b) is the generation of numerical errors at the outlet. However, due to the convective nature of the wave dynamics of falling film flows, these numerical errors are efficiently evacuated and do not accumulate so that only a few nodes are affected near the outlet. In this first case, the matrix to invert at each time step is band diagonal which can be efficiently done using the LU decomposition technique.

In a periodic domain, the closed-flow boundary conditions are written as:

$$
\begin{gathered}
\mathbf{H}(x, 0)=(1,1 / 3)+\mathbf{B}(x), \\
\mathbf{H}(L, t)=\mathbf{H}(0, t), \partial_{t} \mathbf{H}(L, t)=\partial_{t} \mathbf{H}(0, t) .
\end{gathered}
$$

The function $\mathbf{B}(x)$ is a spatial white noise. In this second case, the system matrix is no more band diagonal and a more sophisticated method based on Thomas algorithm was employed.

In practice, we have used a noise amplitude of 0.001 , a spatial mesh size of 0.15 and a time step of 0.01 . The computations were performed on a typical workstation and lasted from a few days to several weeks.

\section{Drag-gravity regime $(\delta<1.5)$}

\subsection{The Nusselt profile as a minimum of viscous dissipation}

The idea that the Nusselt velocity profile could be obtained alternatively by minimizing the viscous dissipation in the film comes from the first papers of Kapitza [15]. It is indeed a general property of Stokes flows with given boundary conditions [2]. We briefly recall here the proof of this result (a similar calculation can be found in [11]).

Let us consider a liquid film of constant thickness $h=h_{N}$ flowing down an inclined plane. The vertical case is singular since the critical Reynolds number for instability is zero: without any loss of generality, we will deal here with the case $\beta<90^{\circ}$. We look for the velocity profile $u(y)$ given the boundary conditions of no slip at the wall $u(0)=0$ and free shear at the interface $u^{\prime}(h)=0$. The viscous dissipation inside the film per unit streamwise length is:

$$
\Phi(u)=\eta \int_{0}^{h}\left(\frac{\partial u}{\partial y}\right)^{2} d y
$$

The differential of $\Phi(u)$ with respect to $u$ is written as:

$$
\Phi(u+\epsilon w)-\Phi(u)=2 \epsilon \eta \int_{0}^{h} \frac{\partial u}{\partial y} \frac{\partial w}{\partial y} d y+O\left(\epsilon^{2}\right)
$$

The boundary conditions for the velocity perturbation $w$ are: $w(0)=0, w^{\prime}(h)=0$. An additional condition arises from the conservation of the total flow rate: $\int_{0}^{h} w(y) d y=0$. Integrating by parts in (6) and using the boundary conditions, we get:

$$
\Phi(u+\epsilon w)-\Phi(u)=-2 \epsilon \eta \int_{0}^{h} w \frac{\partial^{2} u}{\partial y^{2}} d y+O\left(\epsilon^{2}\right)
$$


A necessary and sufficient condition in order to set the differential of $\Phi(u)$ to zero for any function $w(y)$ is: $\frac{\partial^{2} u}{\partial y^{2}}=C \neq 0$. This gives the semi-parabolic Nusselt profile.

\subsection{Short-optimal waves (bound states)}

We examine now the case in the presence of waves and the selection process in the drag-gravity regime. The velocity profile is prescribed to be self-similar to the Nusselt one. This assumption is actually very accurate in this regime for which the amplitude of the waves remains small [14]. The unknown quantity becomes the preferred wavelength or frequency.

\subsubsection{Linear stability}

A necessary condition to observe a wave train at a given period is that this traveling solution is stable with respect to infinitesimal perturbations. The associated linear stability procedure is called a Floquet analysis because the base state is periodic. It is usually written using the moving frame variable $\xi=x-c t$ and put in the form:

$$
\mathbf{H}(\xi, t)=\mathbf{H}_{0}(\xi)+\mathbf{H}_{1}(\xi) e^{i \psi k_{x} \xi+\alpha t}
$$

$\mathbf{H}$ denotes the vector of unknowns $(h, q), \mathbf{H}_{0}$ is the periodic base state with wavenumber $k_{x}, \mathbf{H}_{1}$ is a perturbation amplitude with the same period, $\alpha$ is the complex growth rate. The period of the resulting solution $\mathbf{H}$ is allowed to vary through the parameter $\psi$ which is tuned from 0 (same period) to 0.5 (period doubling).

The stability of the different branches of solutions is wellunderstood thanks to several studies $[7,6,30]$. We recall here the main results on this question. In the terminology introduced by Chang, there are two main wave families. The $\gamma_{1}$ waves show a dominant depression at low wavenumbers and are slower than the temporally most amplified linear waves. These $\gamma_{1}$ waves, as well as the hybrid families $\left(\gamma_{1}^{\prime}, \gamma_{2}^{\prime}, \gamma_{1}^{\prime \prime} \ldots\right)$ are unstable for all wavenumbers: we will therefore not consider them. The $\gamma_{2}$ waves are faster than the temporally most amplified linear waves and show a dominant elevation at low wavenumbers. This wave branch is the only one for which stable waves exist. In the drag-gravity regime, the wavenumbers $k_{x, n}$ of these stable waves form a discrete set of values and vary like $\omega_{c} / n$, with $\omega_{c}$ the oscillation frequency of the capillary waves. The waves synchronize themselves using their capillary radiations. Between these values, the solutions are unstable to subharmonic $(\psi=0.5)$ or sideband $(\psi \ll 1)$ instability modes. At low wavenumbers, this discrete sequence is truncated by the flat-film instability of the substrate $(\psi=0)$.

\subsubsection{Viscous dissipation}

In the drag-gravity regime, the inertial terms are small and we expect the minimum dissipation argument to remain approximately valid. This was speculated by Kapitza [15] and later on by Chang [6] using a different expression for the viscous dissipation. In addition, Trifonov [30] introduced the concept of optimal waves which minimize $\langle h\rangle_{\xi}$ for constant-flux formulation $\langle q\rangle_{\xi}=1 / 3$. We contrast here these different approaches and discuss their ranges of validity.

Figure 2 presents the variations with the frequency of the three energy functionals mentioned above along the $\gamma_{2}$ branch of solutions for $\delta=1$ and $\Gamma=2000$. The expression derived by Kapitza is $\Phi_{1}=9\left\langle\left(\partial_{y} u\right)^{2}\right\rangle_{\xi}=9\left\langle q^{2} / h^{3}\right\rangle_{\xi}$. $\Phi_{1}$ admits three local minima at $f_{1}=8.3 \sim 16.5 / 2 \mathrm{~Hz}, f_{2}=5.4 \sim 16.5 / 3$ $\mathrm{Hz}, f_{3}=4.1 \sim 16.5 / 4 \mathrm{~Hz}$. Chang introduced a more complete expression $\Phi_{2}=9\left\langle\left(\partial_{y} u\right)^{2}+\left(\partial_{x} v\right)^{2}\right\rangle_{\xi}$. These curves $\Phi_{1}(f)$ and $\Phi_{2}(f)$ are superimposed, which indicates that the additional term $\left(\partial_{x} v\right)^{2}$ is negligible in this case. The functional $\Phi_{3}=\langle h\rangle_{\xi}$ considered by Trifonov agrees qualitatively well with the two other ones.

The most general expression for viscous dissipation given by Rayleigh (see for instance [3]) is written as:

$$
\Phi_{4}=\int_{0}^{h}\left\langle 2\left[\left(\partial_{x} u\right)^{2}+\left(\partial_{y} v\right)^{2}\right]+\left[\partial_{y} u+\partial_{x} v\right]^{2} d y\right\rangle_{\xi}
$$

Computations of this functional show similar behaviours, apart from the fact that the curve is reversed with the consequence that the values are always higher than in the case of a smooth film. Physically, it is not clear in which case (wavy or smooth film) viscous dissipation should be the highest. Numerically, we have observed that the equation model and the condition used to compute the waves (constant-flux or constant-thickness) are crucial: viscous dissipation is sometimes found lower in the wavy case [12] or smooth case [6].

Indeed, this question amounts to finding $\min \langle\Phi(h, q)\rangle_{\xi}$ under the constraint $\langle q\rangle_{\xi}=1 / 3$ or $\langle h\rangle_{\xi}=1$. It could be treated under the framework of Lagrange multipliers, but we leave this for future work. For low $\delta$ values, the energy functionals can be expanded around the flat film solution which explains the fact that all these latter show similar behaviours. Some of them may be reversed because of the sign of the coefficients in the expansion. For higher $\delta$ values, we have observed significant differences between them. As a consequence, we do not attempt to discuss the long-time behaviour of the film in the drag-inertia wavy regime using these viscous dissipation functions. Since the inertial terms are no more negligible, it is an open question to know if the minimum viscous dissipation argument remains valid in the drag-inertia regime, or if not which functional to minimize is relevant.

\subsubsection{Direct numerical simulations}

The crucial question is to check that these short-optimal wave solutions are indeed selected by the system when performing direct numerical simulations. Figure 3 compares the traveling-wave optimal profiles (Fig 3a, 3b, 3c) to snapshots from the time-dependent simulations of the model (Fig 3d) with parameter values corresponding to Figure 2. In some parts of the domain, we have found groups of synchronized waves. These groups do not spread over the entire domain but are separated by portions of disordered states. Moreover, the preferred optimal solution among the three ones illustrated in Figure 3 is the one of intermediate frequency $(f=5.2 \mathrm{~Hz})$. This point was addressed in fact recently by Pradas et al. [23] in the draggravity regime. Performing statistics on the separation lengths between the waves, they found peaks around the values corresponding to bound states predicted by the theory. They do not conclude on which solution is the preferred one but their histograms show as well a greater number of waves for intermediate values of the separation length. Interestingly, they observed 


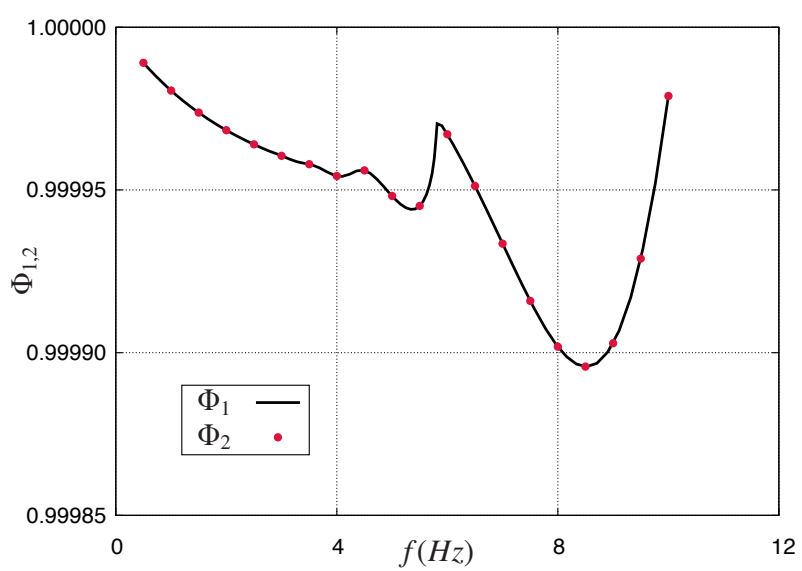

(a)

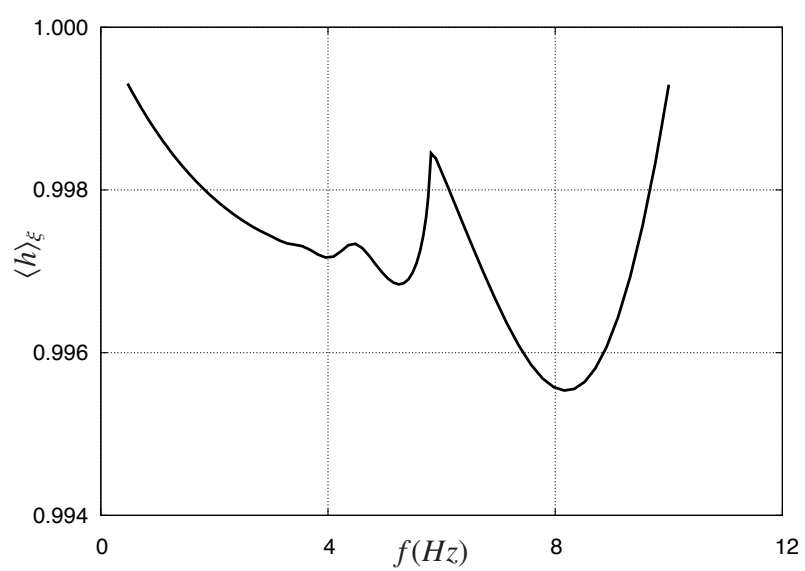

(b)

Figure 2: Variation with the frequency of two energy functionals $\Phi_{i}$ as well as $\langle h\rangle_{\xi}$ along the $\gamma_{2}$ traveling-wave branch of solutions $(\delta=1, \Gamma=2000)$

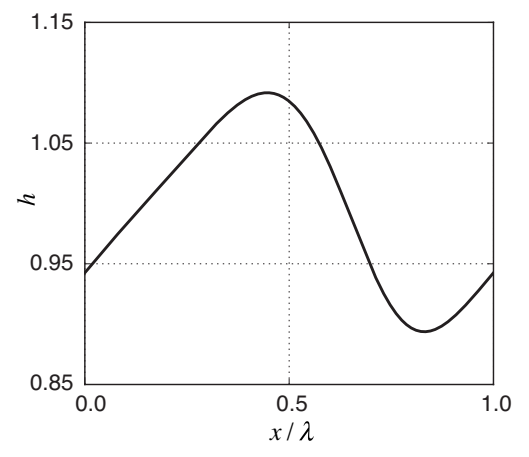

(a) $f=8.2 \mathrm{~Hz}$

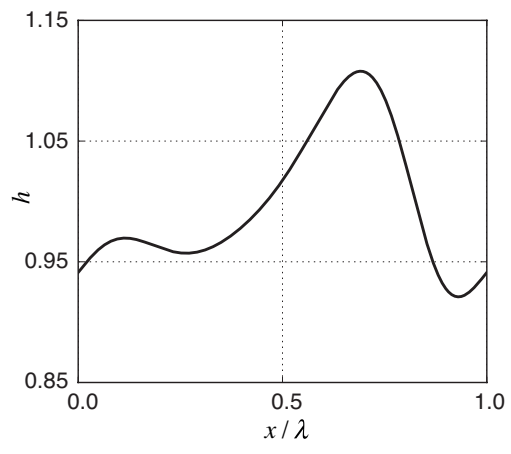

(b) $f=5.2 \mathrm{~Hz}$

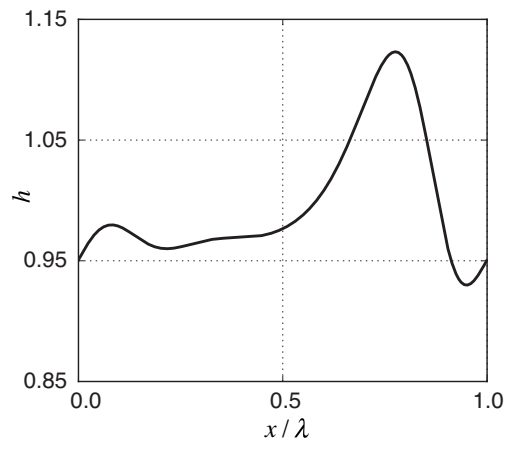

(c) $f=4.0 \mathrm{~Hz}$

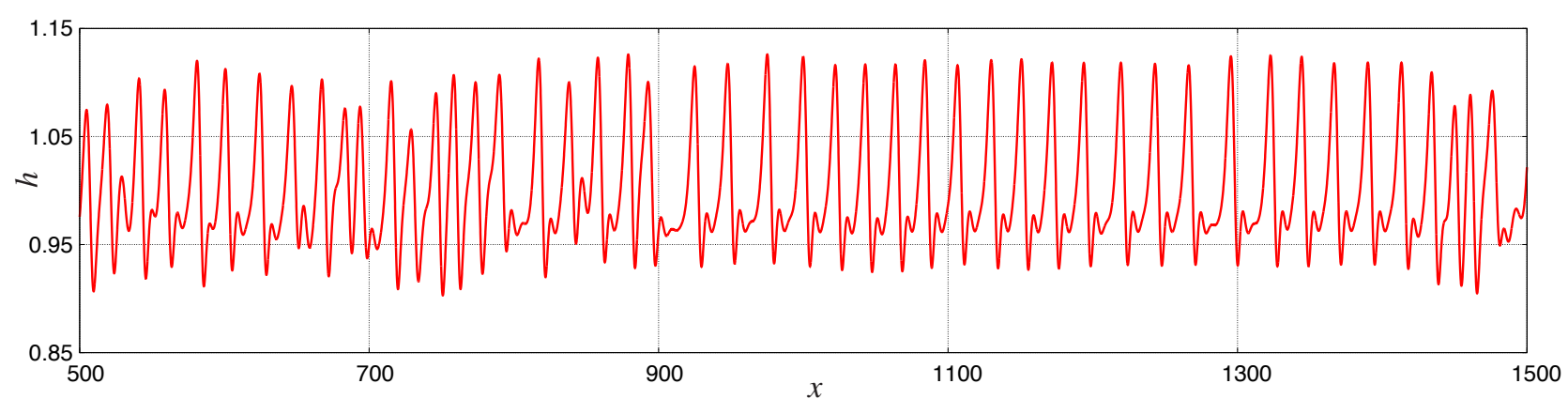

(d)

Figure 3: Short-optimal wave solutions $(\Gamma=2000, \delta=1)$. Figure $3 \mathrm{a}, 3 \mathrm{~b}, 3 \mathrm{c}$ are traveling-wave profiles corresponding to stable periodic waves and to the local minima of Figure 2b. Figure 3d shows a snapshot from a spatio-temporal simulation of the low-dimensional model (1).

also oscillatory behaviour for certain values of the initial separation distance [21].

The selected state is thus not necessarily the one corresponding to the lowest local minimum of viscous dissipation. We conjecture that the selection of the observed local minimum depends on the linear filtering of the inlet signal. We have thus compared the velocity of these short-optimal waves $c_{f \mathrm{~Hz}}$ with the one $c_{s}$ of the spatially most amplified linear waves: $c_{4.0 \mathrm{~Hz}}>c_{5.2 \mathrm{~Hz}}>c_{s}=0.991>c_{8.2 \mathrm{~Hz}}$. As the nonlinear wavemerging process tends to accelerate the flow, the wave velocity tends to increase away from inlet, with the consequence that the slowest optimal-wave solution at $8.2 \mathrm{~Hz}$ cannot be selected.

\section{Drag-inertia regime $(\delta>1.5)$}

\subsection{Open-domain simulations: a new preferred separation}

Let us turn now to the drag-inertia regime. From the point of view of the linear stability of the $\gamma_{2}$ branch of solutions, the situation appears quite different from the drag-gravity regime. As explained by Chang et al. [6], bound states become unstable in this regime. For low values of the wavenumber $k<k_{f}$, the waves are still unstable due to the flat film instability of the substrate but a band of stable wavenumbers appears $k_{f}<k<$ $k_{s}$. Among this continuum of stable solutions, we will investigate which one is more preferred.

Figure 4 shows a snapshot from an open-domain simulation for $\delta=10, \Gamma=20$. After inception, we see the formation of 
large amplitude solitary waves. However, the selection process at work is weaker than in the drag-gravity regime (Figure $3 \mathrm{~d}$ ). Pradas et al. [23] observed also that the pulses lose their boundstate self-organization in this regime. Their distribution functions of the mean-separation distance are quite broad but still show some peak.

Following this idea, we investigate here the shape of these distribution functions and their dependence on the distance from inlet. Figure 5 presents two frequency histograms for the same sets of parameters as in Figure 4, one corresponds to a location near inlet of the white zone $0<x<3000$ (Fig 5a) and the other one to a location close to outlet of the white zone (Fig 5b). We have used a large sample (more than 4000 waves) to achieve good convergence of the statistics. Nevertheless, the former case would require even more waves as it is visibly more sensitive to inlet noise. The two histograms show that there are nearly no waves whose frequency is below $2.5 \mathrm{~Hz}$ or above 15 $\mathrm{Hz}$ as the associated solutions are certainly unstable. The peak around $5 \mathrm{~Hz}$ does not depend much on the spatial location as we shall see later. The distributions have a long tail that decays faster near outlet.

Given these long-tailed distribution shapes, we now compare them with Levy distributions. Indeed, our distribution functions cannot be viewed as a sum of independant events (Gaussian case). There are long-range interactions through the flat film and extreme events which do not have negligible probability: coalescence between the waves. The Levy distribution function that we employ here is the only one as far as we know that can reproduce these features: it has a heavy tail and is independant of the time horizon (stable distribution). Its expression is written as:

$$
P(x)=\sqrt{\frac{c}{2 \pi}} \frac{1}{(x-\mu)^{\alpha}} \exp \left(\frac{-c}{2(x-\mu)}\right)
$$

The parameter $\mu$ controls the mean of the distribution, $c$ is a shape factor and $\alpha$ is the tail exponent (usually $\alpha=3 / 2$ but we stick here the most general expression). In our case, the existence of a cut-off frequency provides a finite variance but Figure 5 shows that the agreement is convincing. It seems that going away from inlet can be modeled increasing the tail exponent $\alpha$. The distribution is becoming more symmetric near outlet and begins to present a more Gaussian shape (the log-log plot of Figure $5 b$ indicates some disagreement with the fitted Levy distribution for the largest frequencies). As we will see later, this process is very slow and difficult to investigate due to computational cost limitations.

\subsection{Traveling-wave solutions: a new minimum of $\langle h\rangle_{\xi}$}

The shape of the traveling-wave solutions of the $\gamma_{2}$ branch is quite different between the drag-gravity and the drag-inertia regime. In the latter case, the waves have a large amplitude and speed. Moreover, the characteristic lengths of their front and back are no more equal but rather scale as: $X_{\text {front }} \sim 1$, $X_{\text {back }} \sim \delta[14,19]$. These properties lead to an important consequence illustrated in Figure 6 . The mean height of the waves along the $\gamma_{2}$ branch of solutions shows a minimum at low frequency values that is not present in the drag-gravity regime. We recall here that the open-flow condition $\langle q\rangle_{\xi}=1 / 3$ is enforced. Similarly to the capillary local minima also visible in
Figure $6 \mathrm{~b}$, this new global minimum arises most probably from the coupling between this condition and the change in scalings explained above. We observe also that, due to the large amplitude of the waves, there is a $10 \%$ variation of $\langle h\rangle_{\xi}$ which is much higher than in the drag-gravity regime (only $0.5 \%$ variation in Figure 2b). The solution associated with this global minimum, whose profile is depicted in Figure $6 \mathrm{a}$, has been called "long-optimal wave" by Trifonov [31]. However, it was not noticed that it exists only in the drag-inertia regime.

\subsection{Selection of the long-optimal waves}

Let us recall briefly the results obtained in the previous sections 4.1 and 4.2. From the open-domain simulations of the model, we have seen that there is some preferred separation between the waves in the drag-inertia regime. The system is clearly not at equilibrium since the frequency distributions vary significantly with the distance from inlet. We have introduced a special category of traveling-wave solutions associated with a new global minimum of the mean height along the $\gamma_{2}$ branch. We make the link here between these results and show that the waves selected by the simulation are indeed these long-optimal solutions. In the two remaining sections of the paper, we will justify physically why these waves are selected and explore the ability of periodic-domain simulations to reach the equilibrium state of the system.

Figure 7 shows the time averaged characteristics of the waves (frequency, mean and maximum height) as functions of the distance $x$ from inlet for the parameter set corresponding to Figure 4 . These three quantities converge towards the optimal values. The convergence is very fast for $\langle h\rangle_{\xi}$ whereas it seems to require a larger distance from inlet for $h_{\text {max }}$ and $f$. The computation of the number of wave-peaks at each position $x$ for a given time window (insert in Figure 7a) demonstrates that coalescence events, though rare, still occur.

Inserts presenting histograms at a location far from inlet $(x=2700)$ have been added to Figures $7 \mathrm{~b}$ and $7 \mathrm{c}$. The distribution of $\langle h\rangle_{\xi}$ shares similarities with the frequency histogram depicted in Figure 5: a long tail with a clear accumulation above $\langle h\rangle_{\xi} \sim 0.8$. For a small part of the sample however, $\langle h\rangle_{\xi}>1$ which indicates that these waves are not close to traveling-wave solutions. The histogram of $h_{\max }$ has a different shape: it is almost symmetric. This effect is probably related to the fact that the waves present a spatial modulation (clearly visible in Figure 4), which will be discussed later in this paper. The histogram of $c$, also displayed in an insert, is similar to the one of $h_{\text {max }}: \Delta c \propto \Delta h_{\text {max }}$, which is a correlation frequently encountered in the literature (see e.g. [18]).

We have checked these results for three other sets of parameters as shown in Table 1. In each case, the optimal quantity (OPT), corresponding to the optimal traveling-wave solution, is compared with the time averaged value computed from the open-domain time-dependent simulations (DNS). As reported earlier, the agreement is almost perfect for $\langle h\rangle_{\xi}$ and convincing for $f$ and $h_{\text {max }}$.

To complete our investigations, we have examined the influence of the parameters $\Gamma$ and $\delta$ on the wavelength of the optimal traveling-wave solutions (Figure 8). As the dimensional frequency $f_{H z}$ depends on the Shkadov time scale and thus on $\Gamma$ and $\delta$, it is more relevant to consider here the non-dimensional 


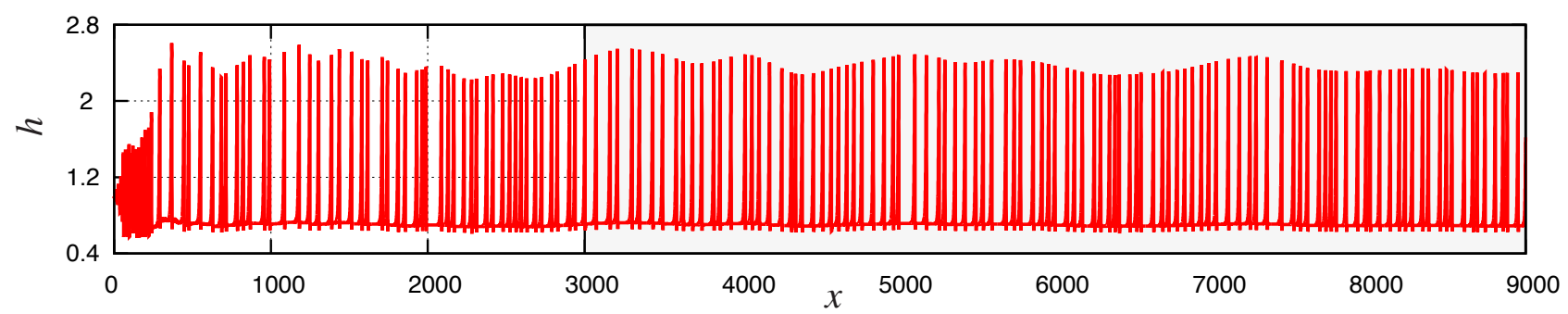

Figure 4: Snapshot from a spatio-temporal simulation of the equations model in an open domain. The parameters are: $\Gamma=20, \delta=10$. The statistics have been performed based on the results of a simulation run on the white zone $0<x<3000$.

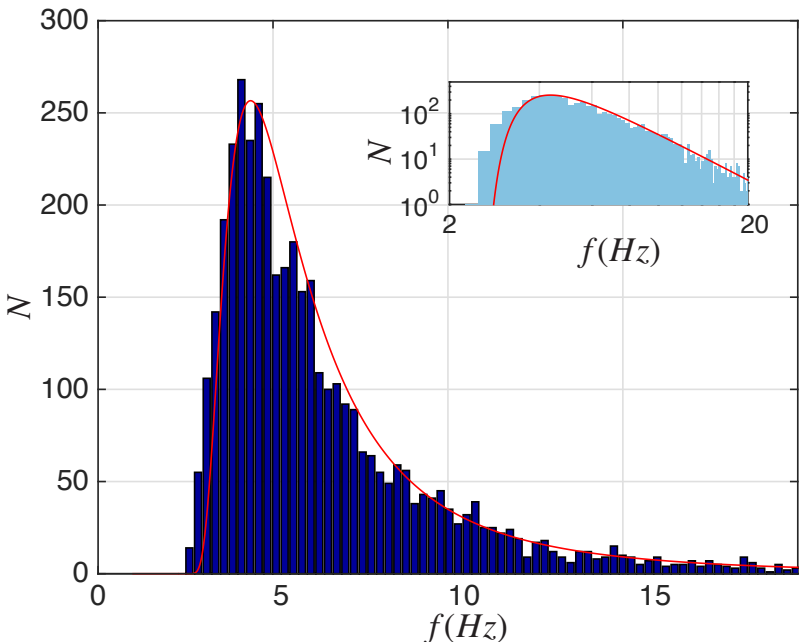

(a) Near inlet, $x=600$

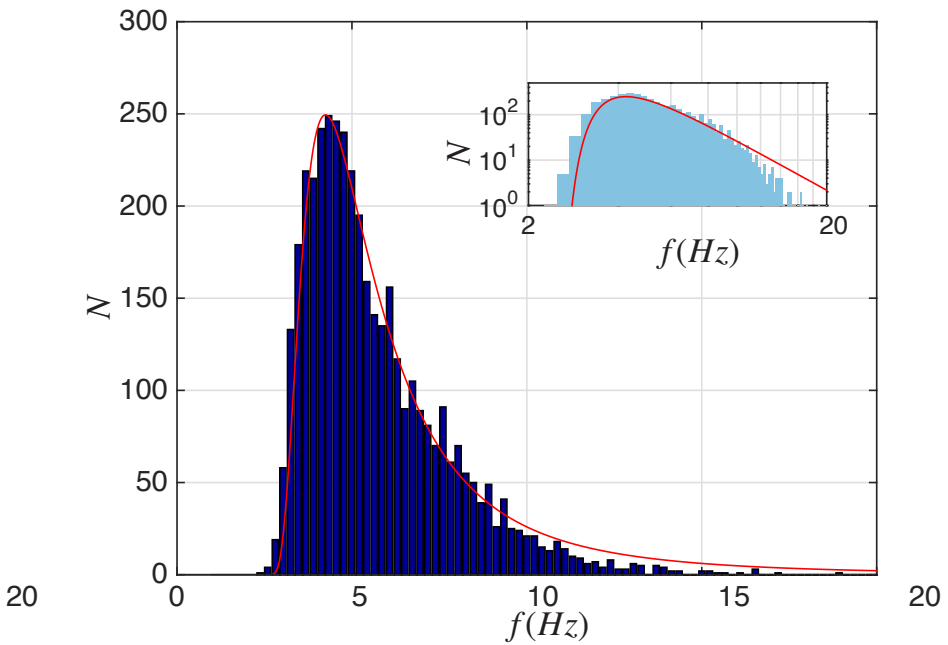

(b) Near outlet, $x=2700$

Figure 5: Frequency distributions of the waves for two different spatial locations along the plane: near inlet, $x=600$ (Fig 5a) and near outlet, $x=2700$ (Fig 5b). The parameters are the same as in Fig 4 and the $x$ location values refer as well to this situation. The red lines are the associated Levy distributions with $c=14$, $\mu=2.3, \alpha=3.4$ in Fig 5a and $\alpha=3.6$ in Fig 5b. The inserts are the same plots in a log-log scale. The total number of waves of the sample is $N_{\text {tot }}=4082$.

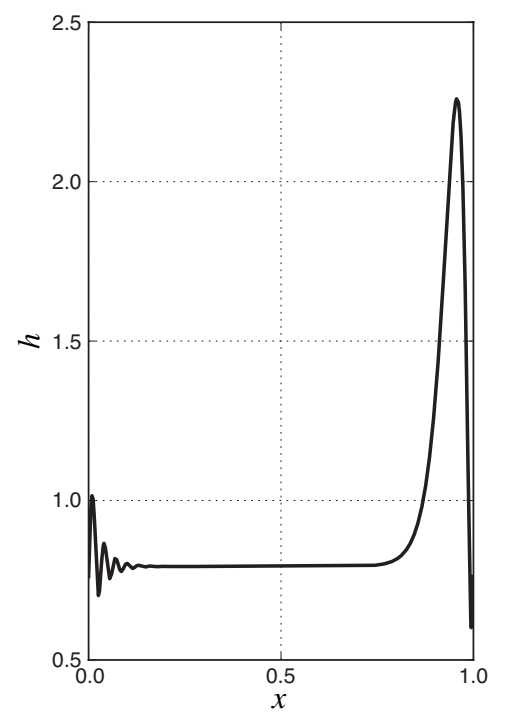

(a)

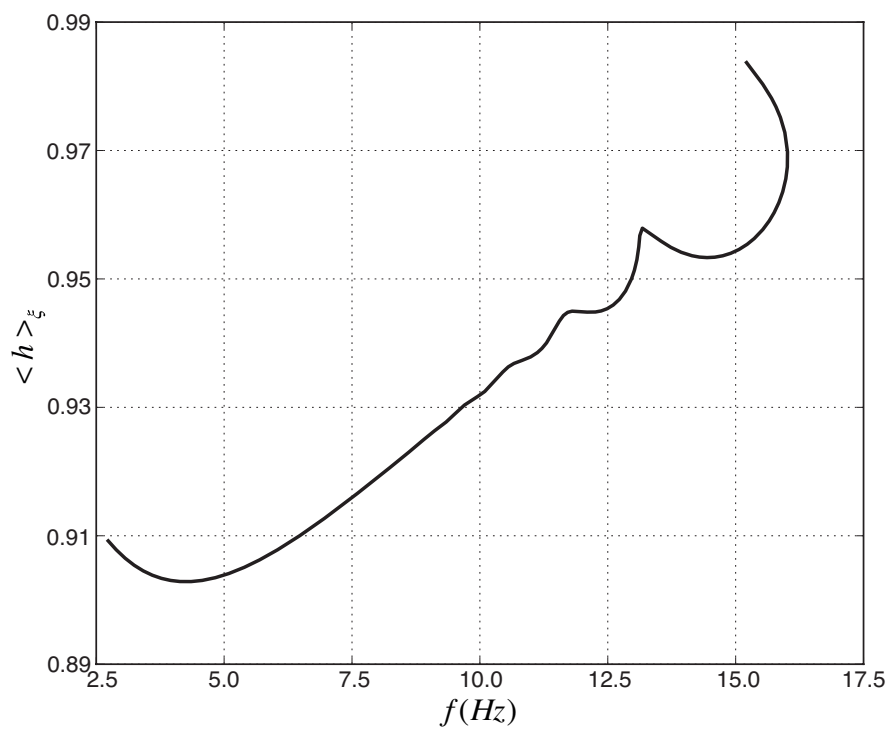

(b)

Figure 6: Optimal profile for $\delta=4.3, \Gamma=500$ (Fig 6a) associated with the global minimum of the mean height along the branch of traveling-wave solutions (Fig 6b) 


\begin{tabular}{|c||c|c||c|c||c|c|}
\hline \multicolumn{1}{|c||}{} & \multicolumn{2}{c||}{$\delta=15, \Gamma=20$} & \multicolumn{2}{c||}{$\delta=4.3, \Gamma=500$} & \multicolumn{2}{c|}{$\delta=4.3, \Gamma=20$} \\
\hline & DNS & OPT & DNS & OPT & DNS & OPT \\
\hline \hline$f_{H z}$ & 5.88 & 5.63 & 4.67 & 4.27 & 3.69 & 3.53 \\
\hline$\langle h\rangle_{\xi}$ & 0.860 & 0.861 & 0.903 & 0.903 & 0.906 & 0.905 \\
\hline$h_{\max }$ & 2.46 & 2.42 & 2.33 & 2.26 & 2.30 & 2.23 \\
\hline
\end{tabular}

Table 1: Time averaged characteristics of the waves near outlet (DNS) and comparison with the optimal values (OPT) for three sets of $(\delta, \Gamma)$. The total number of waves of the sample is: $N_{\text {tot }}=840$.

wavelength $\lambda_{x}$. As explained before, this quantity depends on the relative importance of the different parts of the wave profile (tail, front, capillary waves). For $\delta>20, \lambda_{x}$ is independant of $\Gamma$ and scales as $\lambda_{x} \propto \delta$ : this is consistent with the fact that most of the domain is occupied by the wave tail. The rest of the curve, especially the resonance peak around $\delta=2$, expresses the fact that the contributions of the different parts of the wave profile compete with each other.

\subsection{Discussion}

In this section, we address the following question: in the drag-inertia regime, why should the "optimal" traveling-wave solutions of minimum mean height be selected in the opendomain simulations? We readily reject two possible explanations: (i) that these waves are the most stable, or (ii) that they correspond to a minimum of viscous dissipation. The former argument cannot be invoked since $\gamma_{2}$ traveling-wave solutions present a band of marginally stable waves in the drag-inertia regime. The latter one is not a good candidate either for at least two reasons. Numerically, we have been unable to obtain any significant trends because it depends a lot on precise characteristics of the waves as well as velocity field representation. Moreover, it is not clear physically if it should apply for $\delta>1.5$ as inertia is no more negligible in this regime.

The explanation we suggest is that the mean thickness has no alternative but to decrease in an irreversible manner toward a global minimum since the waves are faster than any infinitesimal disturbance. This result was outlined by Chang and Demekhin [4]. Using the generalized Kuramoto-Sivashinsky equation as well as the Kapitza-Shkadov model, they found that the solitary waves escape the wave packet for $\delta>1$ which corresponds roughly to the limit between the drag-gravity and the drag-inertia regime. Mass drainage can then occur only backwards which imply that the temporal mean thickness has to decrease with the distance to the inlet. It may occur however that the waves stay trapped in the local minima at high values of the Kapitza number (Figure 6b).

To illustrate the fact that mass drainage occurs only backwards, we look at the dynamics of the waves in a frame moving at the optimal wave speed. Figure 9 presents the evolution of the mean thickness over such a moving frame as function of the distance $x$ from inlet. Figure 10 is a spatio-temporal diagram for $1900<x<2800$ (same simulation run). We have chosen a window width of three times the optimal wavelength. This value $\left(\Delta x_{f}=165\right)$ is the result of the following compromise: it is much lower than the total length of the domain $(\Delta x=3000)$ but sufficiently high so that to attenuate the effect of waves re-

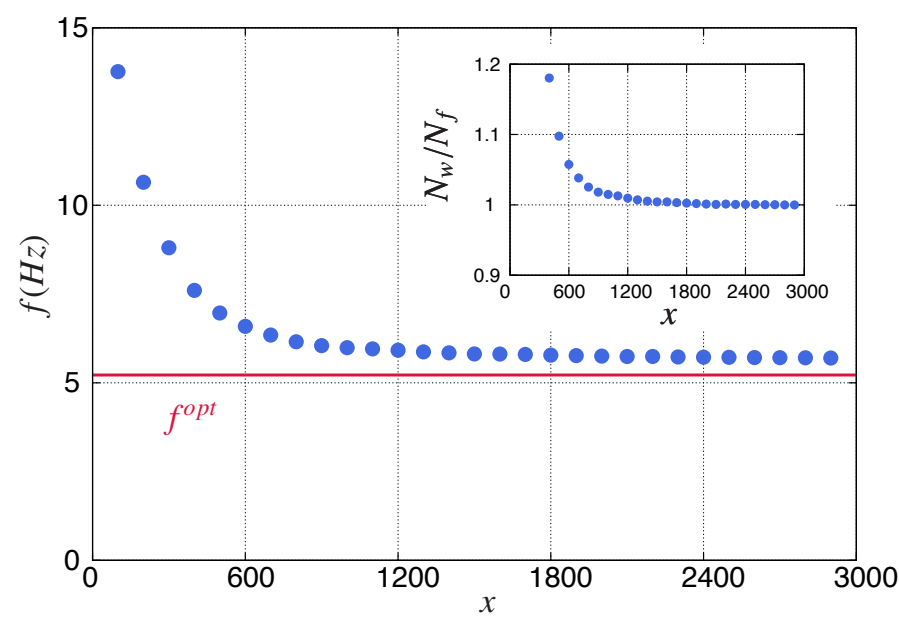

(a)

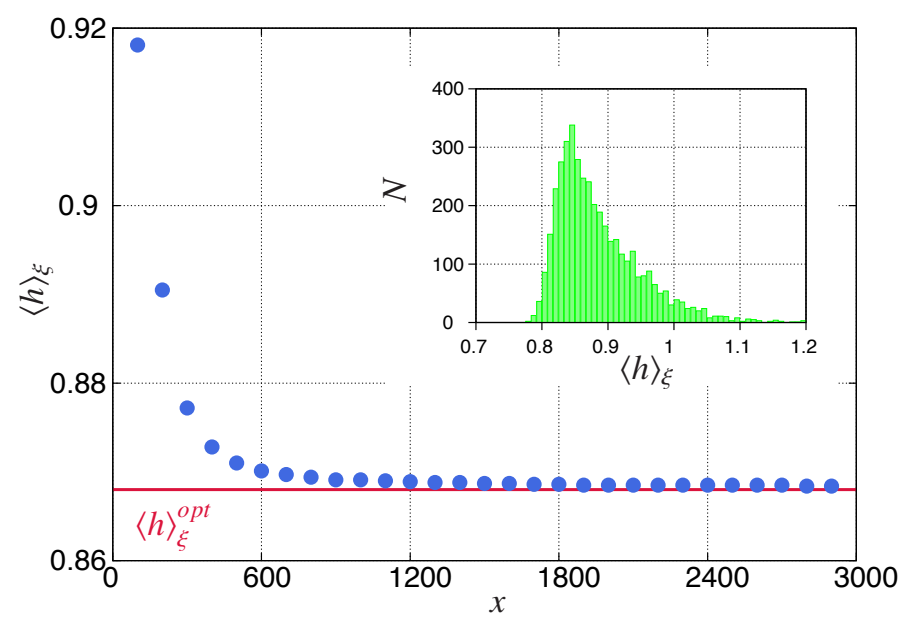

(b)

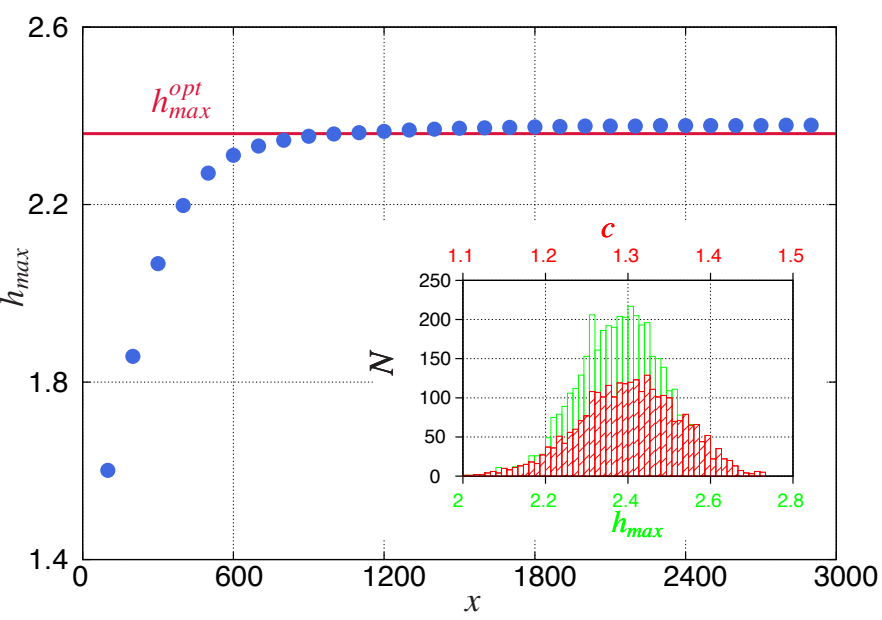

(c)

Figure 7: Time averaged characteristics of the waves as functions of the distance $x$ from inlet and comparisons with the optimal values (parameter values are $\delta=10, \Gamma=20$ ). Total number of waves for the construction of the histograms: $N_{\text {tot }}=4082$. (a): Frequency. In insert: number of waves for a given time window $N_{w}$ divided by its value $N_{f}$ at $x=3000$. (b): Mean height and histogram showing the associated distribution for $x=2700$. (c): Maximum film thickness and distribution in insert $(x=2700)$. The insert presents also the distribution of the celerity of the waves (hatched bars ; $x=2500, N_{t o t}=2816$ ). 


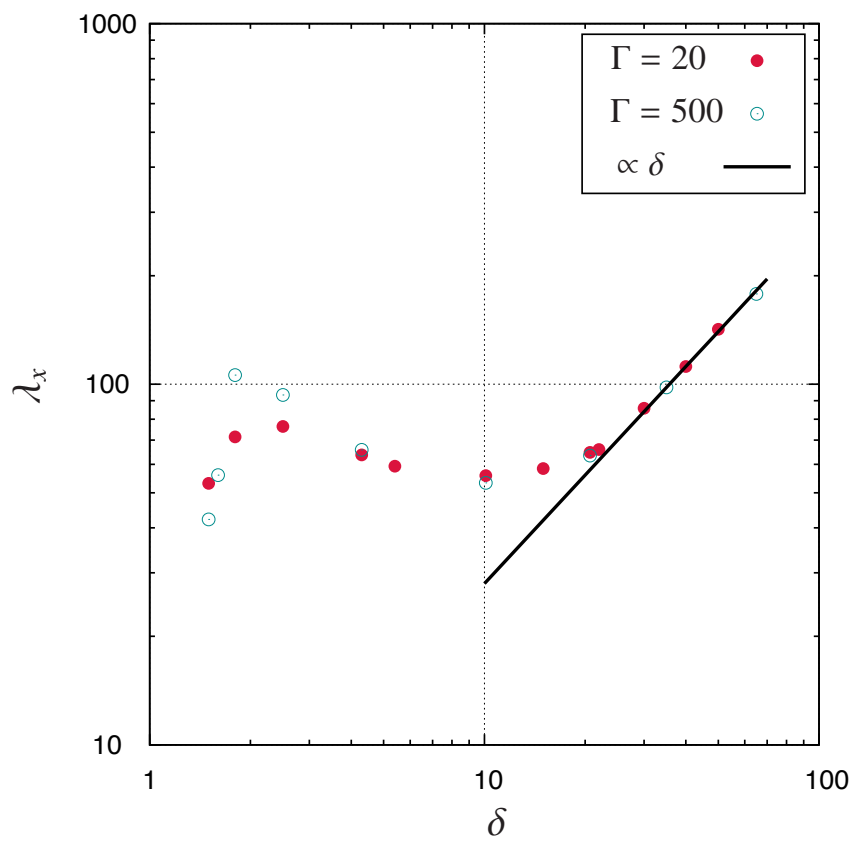

Figure 8: Non-dimensional optimal wavelength $\lambda_{x}$ obtained from the travelingwave solutions as functions of the reduced Reynolds number $\delta$ for two values of the Kapitza number $(\Gamma=20, \Gamma=500)$. The line shows the scaling law at $\delta>20$.

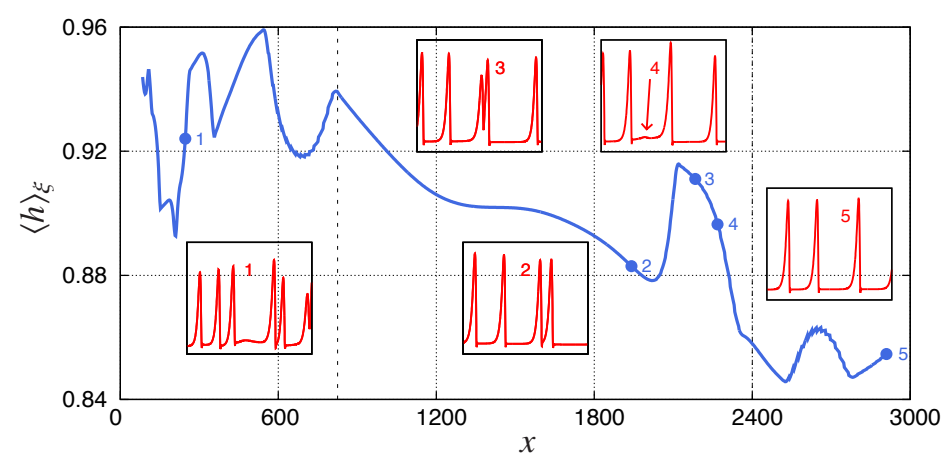

Figure 9: Mean thickness $\langle h\rangle_{\xi}$ over a sliding window as function of the distance $x$ of its center from inlet $(\Gamma=20, \delta=10)$. The sliding window is moving at the optimal wave speed and its width is three times the optimal wavelength. Wave profiles for five specific points are shown in inserts. Vertical dashed lines separate three distinct regimes: the entry zone $(x<800)$, the zone where the waves accelerate and coalesce $(800<x<2400)$ and a quasi-synchronized zone $(x>2400)$.

spectively going in (I) or out $(\mathrm{O})$ of the window. Nevertheless, these latter effects are still visible around $x=2100$ (I), 2200 (O), $2550(\mathrm{I}), 2750(\mathrm{O})$ : they correspond to an increase, respectively a decrease, of the mean thickness over the sliding window. The results show that, after an entrance zone $(x<800)$, the mean thickness of the film decreases as the waves accelerate and coalesce with each other $(800<x<2400)$. Eventually, the system stabilizes into a quasi-stationary state $(x>2400)$ consisting in three waves moving roughly at the optimal wave speed. At each coalescence event, some mass is rejected at the wave back and exits the window as Figure 10 clearly outlines.

Let us compare now our analysis with the one by Chang et al. [5]. Using the Kapitza-Shkadov model, they introduced a

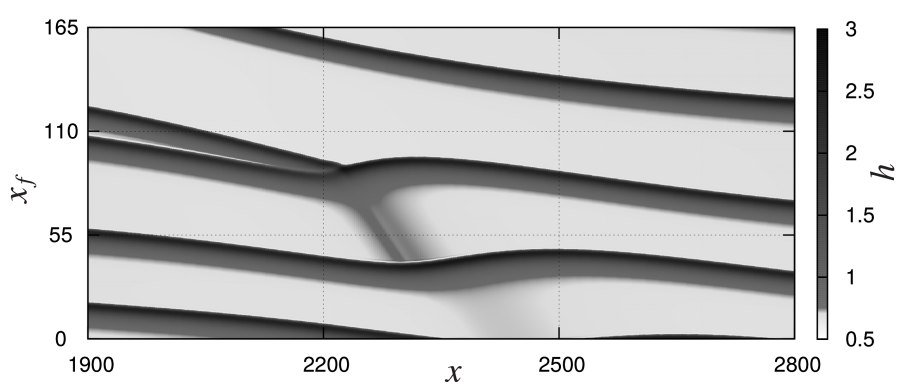

Figure 10: Spatio-temporal plot around the coalescence event corresponding to specific point $\mathrm{n}^{\circ} 4$ in Figure 9. The coordinate $x$ refers to the center location of the sliding window as in Figure 9, $x_{f}$ is the position within the sliding window and $h$ is the thickness of the film.

distinction between two kind of traveling-wave solutions: small reference waves and large excited waves resulting from the coalescence of two small ones. They derived an analytical expression for the coalescence time between these two kinds of pulses and, making this quantity tend to infinity, theoretically deduced the final spacing between the waves (equation (25) of their paper). The crucial point of their analysis relies on the fact that there are as many small pulses as large pulses due to the subharmonic instability at wave inception. It is interesting to point out that their expression of the separating distance is proportional to $\delta$ as in agreement with our observations at large values of $\delta$ (see Figure 8). However, their results do not agree quantitatively with ours which may be due to the assumptions and the type of model they used.

\subsection{Periodic-domain simulations}

In the last part of this paper, we are going to contrast qualitatively our observations for time-dependent simulations in an open domain with similar ones conducted in a periodic domain. This analysis is motivated by the prohibitive cost of simulations in extended domains. Figure 4 points out the very slow decay of a remaining spatial modulation of the wavetrain exiting the open-flow domain. The value of this decay coefficient can be related to the analysis by Chang et al. [4]. Indeed, the spectrum of solitary pulses contains both a discrete and a continuous part. Using an exponentially weighted space, the continuous part can be shifted which brings back the anaylsis of the mass drainage dynamics to a single new eigenvalue: the resonance pole [13]. Even though Chang et al. used the Kapitza-Shkadov model, this argument should remain valid for the current WRIBL model.

The evolution of the wavetrain modulations can be investigated by performing very long-time simulations in a sufficiently extended periodic domain as the temporal evolution of the wavetrain shall be similar to its evolution in an open-flow system. To illustrate the decay of the wavetrain modulation, we report the amplitude of the waves as a function of time in the middle of the domain for $\delta=10, \Gamma=20$ (Figure 11). For small domain lengths (Figure 11a), temporal modulations of the film are nearly harmonic as the number of waves in the domain is small and a final state of the wave organization is reached with a reasonable computational cost. When increasing the size of the domain, the modulations have a broader frequency content (Figures 11b,11c). However, it seems that the attenuation of the first Fourier coefficient does not depend on the domain size. A 
fitting with an exponential $e^{-\gamma t}$ gave us: $\gamma=(4.5 \pm 0.5) .10^{-5}$. Moreover, this spatio-temporal modulation does not travel at the same speed as the waves as shown in the spectrum in insert of Figure 11a.

The time required to relax the entire dynamics of the film to an array of waves of constant amplitude can be estimated from $\gamma$. For the discussed simulation, one gets $t_{f} \sim 5.10^{4}$ (nondimensional units). To transpose this value to the open-domain case, the length required to get access to the final state would be roughly: $x_{f} \sim c \times t_{f}=6.5 \times 10^{4}(c \sim 1.3$ is the mean value of the velocity of the waves). This is impossible to achieve for computing cost reasons.

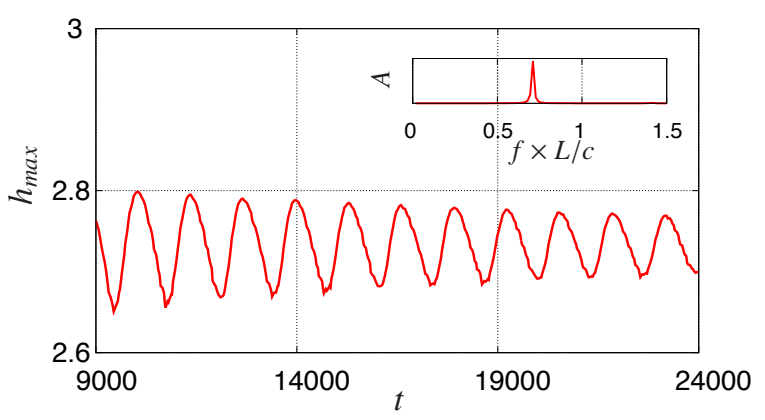

(a) $L=1500$

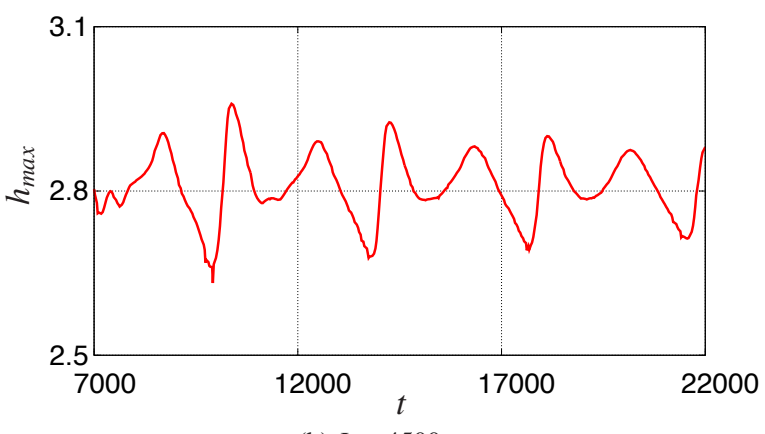

(b) $L=4500$

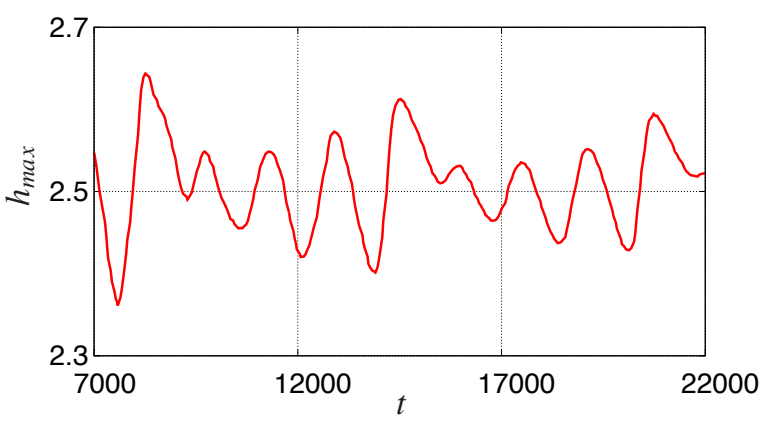

(c) $L=6000$

Figure 11: Influence of the domain length $L$ on results of periodic-domain computations $(\delta=10, \Gamma=20)$. In each case, the amplitude of the waves $h_{\max }$ is plotted as a function of time $t$ for a spatial location $x$ which is the middle of the domain. The signal looks continuous due to the large number of waves by pseudo-period (the period of the waves is much smaller than the period of the modulation). Frequency spectrum in also plotted in the insert of Figure 11a.

From what we have discussed so far, it seems that computations in periodic domains of short length $L$ are well adapted to get the final spacing $\lambda_{f}$ of the waves. However, as illustrated in Figure 12, this spacing is discretized by the boundary conditions: $\lambda_{f}=L / N_{w}$, with $N_{w}$ the number of waves in the domain

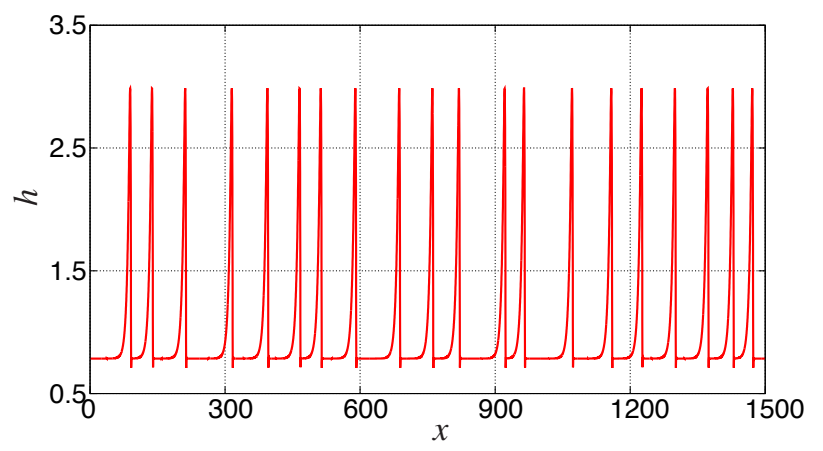

(a) $N_{w}=20$

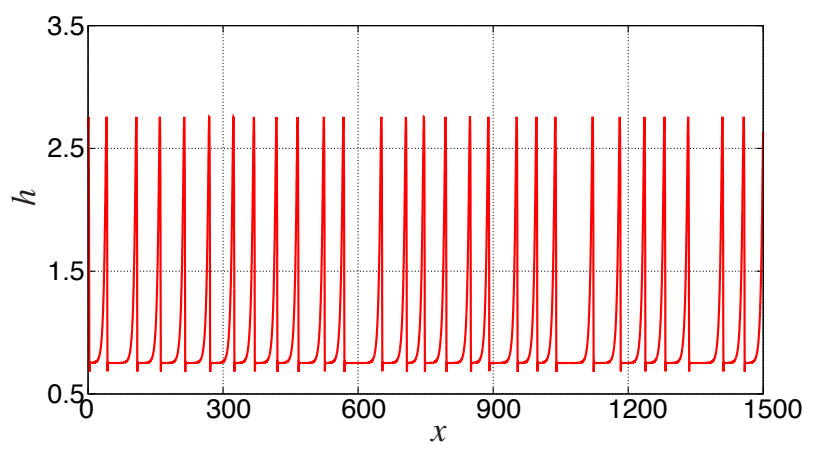

(b) $N_{w}=28$

Figure 12: Final state of periodic-domain simulations $(\delta=10, \Gamma=20)$ for a short domain length $(L=1500)$ : the number of waves $N_{w}$ is different for each run.

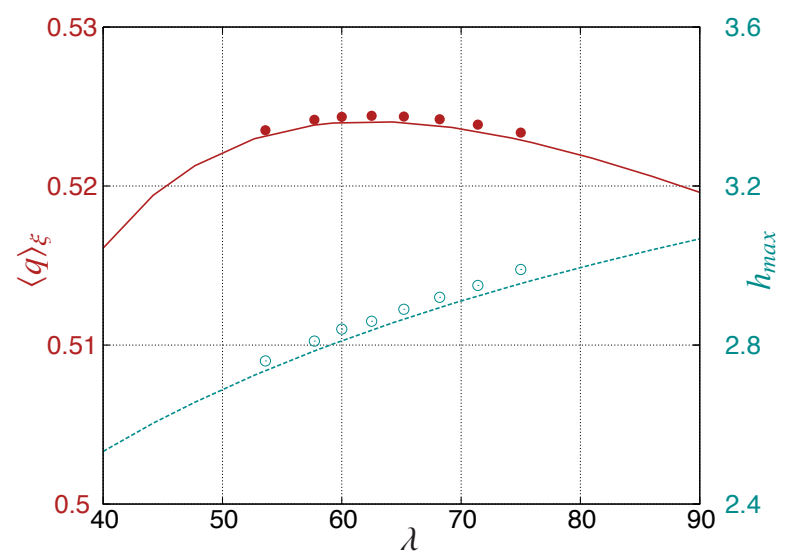

Figure 13: Mean flow rate $\langle q\rangle_{\xi}$ (filled symbols, continuous line) and maximum thickness (open symbols, dashed line) in function of the wavelength along the traveling-wave branch (lines) and several final values obtained from the periodic-domain simulations (circles). The parameters are the same as in Figure 12 .

at the end of the simulation. Thus, given that the initial condition is a random noise, we do not get the same value of $N_{w}$ at the end of each run of the simulation (Figures 12a,12b). It is therefore not possible to do statistics aggregating different simulation runs. Increasing the size of the domain could minimize this effect but would lead back to computing cost issues. Interestingly, even if the amplitude of the waves is constant at the end of the simulation, the spacing between them is not strictly constant: this may be the consequence of the presence of subsidiary homoclinic orbits (several waves moving together with a separation between them not equal to the optimal one). 
These simulations cannot reproduce also the mean height reduction as the total mass is fixed. However, the selected solution corresponds then to the one of maximum mean flow rate, as can be seen in Figure 13. Performing an averaging over 8 simulation runs in order to filter the discretization effect imposed by the boundary conditions, we have indeed obtained $\langle q\rangle_{\xi}=0.524$ which corresponds exactly to the maximum value along the traveling-wave branch.

\section{Conclusion}

In this article, the late time dynamics of solitary waves in a vertically falling liquid film subjected to inlet white noise has been investigated numerically. We have employed for that purpose a reduced set of equations which quantitatively mimics the dynamics of the film as shown by comparisons to experiments and DNSs in e.g. [14, 30] (Ruyer-Quil-Manneville model). In the drag-gravity regime $(\delta<1.5)$, it is already known that the waves synchronize themselves into bound states through the interaction of their back tails with the undulatory front tails (capillary ripples). In contrast, the results we have presented here in the drag-inertia regime $(\delta>1.5)$ are novel. Open-domain simulations show an irreversible decrease of the mean thickness of the film towards its minimum value along the corresponding traveling-wave branch of solutions. The mean characteristics of the waves tend then to the one of these optimal solutions. This convergence process is very slow due to a persistent spatial modulation of the waves. When performing periodic-domain simulations, we obtain similar trends except for the fact that it is now the mean flow rate which increases to its maximal value. Although the simulations suffer from finite-size effects, they allow to reach a fully converged final state. The separation distance between the waves is not found then to be strictly constant due to the possible existence of subsidiary homoclinic orbits.

We believe that these results shall prove to be useful for industrial applications since they allow to predict directly the mean characteristics of the waves in noisy films far from inlet. Further developments include verifications using direct numerical simulations of the basic equations, extension to the $3 \mathrm{D}$ case and accounting for heat and mass transfer. These optimal waves are favorable to diffusive transfers as the effective resistance of diffusion is the harmonic mean film thickness. It would be very interesting to see if this is also the case for convective transfers due to fluid recirculation and mixing in the main hump.

The authors thank Georg Dietze and Nicolas Rimbert for useful discussions, Air Liquide for financial support and EPFLLFMI in which part of this work was performed for hospitality. C. Ruyer-Quil acknowledges financial support from Institut Universitaire de France.

\section{References}

[1] N. Balmforth, S. Mandre, Dynamics of roll waves, Journal of Fluid Mechanics 514 (2004) 1-33.

[2] G. Batchelor, An Introduction to Fluid Dynamics, Cambridge University Press ed., 1974.

[3] R. Bird, W. Stewart, E. Lightfoot, Transport Phenomena, Wiley, NewYork, 1960.

[4] H.-C. Chang, E. Demekhin, Complex wave dynamics on thin films, Elsevier Scientific, 2002.
[5] H.-C. Chang, E. Demekhin, E. Kalaidin, Y. Ye, Coarsening dynamics of falling films solitary waves, Physical Review E 54 (2), 1996.

[6] H.-C. Chang, E. Demekhin, D. Kopelevich, Nonlinear evolution of waves on a vertically falling film, Journal of Fluid Mechanics 250 (1993) 433480.

[7] M. Cheng, H.-C. Chang, Competition between subharmonic and sideband secondary instabilities on a falling film, Phys. Fluids 7 (1) (1995) 34-54.

[8] E. Doedel, AUTO07p continuation and bifurcation software for ordinary differential equations, Montreal Concordia University (2008).

[9] C. Elphick, G. Ierley, O. Regev, E. Spiegel, Interacting localized structures with Galilean invariance, Physical Review A 44 (2) (1991) 1110.

[10] D. Frisk, E. Davis, The enhancement of heat transfer by waves in stratified gas-liquid flow, Int. J. Heat Mass Transf. 15 (1972) 1537-1552.

[11] W. Horne, K. Karamcheti, Extrema Principles of Entropy Production and Energy Dissipation in Fluid Mechanics (1988).

[12] M. Islam, A. Miyara, Numerical investigation of viscous dissipation in wavy falling liquid film, in: Proceedings of the 13th Asian Congress of Fluid Mechanics, 2010.

[13] E. Kalaidin, Spectral properties of nonlinear solitary waves on the surface of thin liquid films, Doklady Physics 51 (5) (2006) 276-278.

[14] S. Kalliadasis, C. Ruyer-Quil, B. Scheid, M. Velarde, Falling liquid films, Springer, 2012.

[15] P. Kapitza, Wave flow of thin layers of a viscous fluid : I. Free flow, in: Collected papers of P.L. Kapitza (1965), Pergamon (Oxford), 1948, pp. 662-689.

[16] T. Kawahara, S. Toh, Pulse interactions in an unstable dissipativedispersive nonlinear system, Physics of Fluids 31 (8) (1988) 2103.

[17] I. Kliakhandler, Inverse cascade in film flows, Journal of Fluid Mechanics 423 (2000) 205-225.

[18] J. Liu, J. P. Gollub, Solitary wave dynamics of film flows, Phys. Fluids 6 (1994) 1702-1712.

[19] T. Ooshida, Surface equations of falling film flows with moderate Reynolds number and large but finite Weber number, Phys. Fluids 11 (1999) 3247.

[20] S. Portalski, A. Clegg, Interfacial area increase in rippled film flow on wetted wall columns, Chemical Engineering Science 26 (1971) 773-784.

[21] M. Pradas, S. Kalliadasis, P.-K. Nguyen, V. Bontozoglou, Bound-state formation in interfacial turbulence: direct numerical simulations and theory, Journal of Fluid Mechanics 716 (R2), 2013.

[22] M. Pradas, S. Kalliadasis, D. Tseluiko, Binary interactions of solitary pulses in falling liquid films, IMA J. Appl. Math. 77 (2012) 408-419.

[23] M. Pradas, D. Tseluiko, S. Kalliadasis, Rigorous coherent-structure theory for falling liquid films: Viscous dispersion effects on bound-state formation and self-organization, Physics of Fluids 23 (4) (2011) 044104.

[24] A. Rastaturin, E. Demekhin, E. Kalaidin, Optimal regimes of heat-mass transfer in a falling film, Journal of Non-Equilibrium Thermodynamics 31 (1) (2006) 1-10.

[25] W. Rohlfs, B. Scheid, Phase diagram for the onset of circulating waves and flow reversal in inclined falling films, Journal of Fluid Mechanics 763 (2015) 322-351.

[26] B. Scheid, C. Ruyer-Quil, U. Thiele, O. A. Kabov, J. C. Legros, P. Colinet, Validity domain of the Benney equation including the Marangoni effect for closed and open flows., J. Fluid Mech. 527 (2005) 303-335.

[27] V. Shkadov, Wave flow regimes of a thin layer of viscous fluid subject to gravity, Izv. Akad. Nauk SSSR, Mekh. Zhidk. Gaza 1 (1967) 43-51.

[28] V. Ya. Shkadov, Solitary waves in a layer of viscous liquid, Izv. Akad. Nauk SSSR, Mekh. Zhidk Gaza 1 (1977) 63-66.

[29] M. K. Smith, The mechanism for the long-wave instability in thin liquid films, J. Fluid Mech. 217 (1990) 469-485.

[30] Y. Trifonov, Stability and bifurcations of the wavy film flow down a vertical plate: the results of integral approaches and full-scale computations, Fluid dynamics research 44 (3) (2012) 031418.

[31] Y. Trifonov, Stability of the wavy film falling down a vertical plate : The DNS computations and Floquet theory, International Journal of Multiphase Flow 61 (2014) 73-82.

[32] D. Tseluiko, S. Kalliadasis, Weak interaction of solitary pulses in active dispersive-dissipative nonlinear media, IMA J. Appl. Math. 79 (2014) 274-299.

[33] G. B. Whitham, Linear and Nonlinear Waves, John Wiley \& Sons New York, 1974.

[34] P. Yoshimura, T. Nosoko, T. Nagata, Enhancement of mass transfer into a falling laminar liquid film by two-dimensional surface waves - Some experimental observations and modeling, Chemical Engineering Science 51 (8) (1996) 1231-1240. 